\title{
STARLIKENESS OF DOUBLE INTEGRAL OPERATORS
}

\author{
RASOUL AGHALARY AND SANTOSH JOSHI
}

\begin{abstract}
In this paper we investigate starlikeness of double integral operators by using second-order differential inequalities. We shall give some interesting conditions for $f(z)$ defined by double integral operators to be starlike of order $\beta$.
\end{abstract}

\section{INTRODUCTION}

Let $\mathcal{H}$ denote the class of analytic functions in the open unit disc $\Delta=$ $\{z \in \mathbb{C}:|z|<1\}$ and $\mathcal{A}_{n}$ denote the class of all functions $f$ in $\mathcal{H}$ such that $f$ has the form

$$
f(z)=z+\sum_{k=n+1}^{\infty} a_{k} z^{k}
$$

where $n \in \mathbb{N}$ is fixed. Set $\mathcal{A}:=\mathcal{A}_{1}$. For a positive integer $n$ and $a \in \mathbb{C}$, we define the following class of analytic functions:

$$
\mathcal{H}[a, n]=\left\{f \in \mathcal{H}: f(z)=a+a_{n} z^{n}+a_{n+1} z^{n+1}+\cdots, z \in \Delta\right\} .
$$

Also let $S^{*}(\alpha)$ denote the familiar class of functions in $\mathcal{A}$ that are starlike of order $\alpha$. It is well known that this class is analytically characterized by

$$
\operatorname{Re}\left(\frac{z f^{\prime}(z)}{f(z)}\right)>\alpha \quad(z \in \Delta, 0 \leq \alpha<1) .
$$

For $f$ and $g$ in $\mathcal{H}$, a function $f$ is subordinate to $g$, written as $f(z) \prec g(z)$, if there is an analytic function $\omega$ satisfying $\omega(0)=0$ and $|\omega(z)|<1$, such that $f(z)=g(\omega(z), z \in \Delta$. If $g$ is univalent in $\Delta$, then $f$ is subordinate to $g$ which is equivalent to $f(\Delta) \subseteq g(\Delta)$ and $f(0)=g(0)$.

Many authors have given sufficient conditions for starlikeness of analytic functions (see [1], [2], [6], [7], [8]). In [4], Kuroki and Owa proved the following results:

2010 Mathematics Subject Classification. Primary: 30C45; Secondary: 30C80.

Key words and phrases. Starlike functions, differential subordination, integral operators. 
Theorem 1.1. Let $f(z) \in \mathcal{A}_{n}$ and let $0 \leq \alpha<n$ and $0 \leq \beta<1$. If $f(z)$ satisfies

$$
\left|z f^{\prime \prime}(z)-\alpha\left(f^{\prime}(z)-1\right)\right|<\frac{(n+1)(1-\beta)(n-\alpha)}{n+1-\beta}
$$

then $f(z)$ is starlike of order $\beta$ in $\Delta$.

Corollary 1.1. Let a function $g(z) \in \mathcal{H}$ satisfy

$$
|g(z)| \leq \frac{(n+1)(1-\beta)(n-\alpha)}{n+1-\beta},
$$

for some $0 \leq \alpha<n$ and $0 \leq \beta<1$. Then the function $f(z)$ given by

$$
f(z)=z+z^{n+1} \int_{0}^{1} \int_{0}^{1} g(r s z) r^{n-\alpha-1} s^{n} d r d s
$$

is starlike of order $\beta$ in $\Delta$.

We note that by setting $\beta=0$ in Theorem 1.1 and Corollary 1.1 we obtain the results of Miller and Mocanu [5].

However the case $\alpha=n$ and $\alpha \in \mathbb{C}$, which does produce a slightly different implication, have not been discussed in [4] and [5]. In this paper we aim to investigate such cases.

For establishing our results, we need the following lemma concerning differential subordinations.

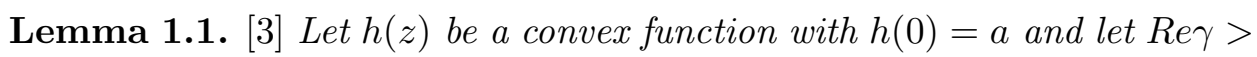
0 . If $p(z) \in \mathcal{H}[a, n]$ and

$$
p(z)+\frac{z p^{\prime}(z)}{\gamma} \prec h(z),
$$

then

$$
p(z) \prec q(z) \prec h(z),
$$

where

This result is sharp.

$$
q(z)=\frac{\gamma}{n z^{\gamma / n}} \int_{0}^{z} h(t) t^{\gamma / n-1} d t
$$

\section{Main Results}

Theorem 2.1. Let $f(z)=z+a_{n+1} z^{n+1}+\cdots \in \mathcal{A}_{n}$ be such that $\left|a_{n+1}\right|<$ $1 /(n+1)$. Also suppose that $0<\lambda \leq 1-(n+1)\left|a_{n+1}\right|$. If $f(z)$ satisfies

$$
\left|z f^{\prime \prime}(z)-n\left(f^{\prime}(z)-1\right)\right|<\lambda
$$

then $f(z)$ is starlike of order $\beta$ in $\Delta$, where

$$
\beta=\frac{(n+2)\left[1-(n+1)\left|a_{n+1}\right|-\lambda\right]}{(n+2)-(n+2)\left|a_{n+1}\right|-\lambda} .
$$


Proof. We can rewrite the inequality (2.1) in terms of subordination as

$$
z f^{\prime \prime}(z)-n\left(f^{\prime}(z)-1\right) \prec \lambda z .
$$

If we set

$$
p(z):=f^{\prime}(z)-(n+1) \frac{f(z)}{z}=-n+a_{n+2} z^{n+1}+\cdots \in \mathcal{H}[-n, n+1],
$$

then $p(z)$ is analytic in $\Delta, p(0)=-n$ and $p^{(k)}(0)=0$ for $k=1,2, \ldots, n-1$. Further, (2.3) is seen to be equivalent to

$$
p(z)+z p^{\prime}(z) \prec-n+\lambda z .
$$

Applying Lemma 1.1 to (2.5), we obtain

$$
p(z) \prec \frac{1}{(n+1) z^{1 /(n+1)}} \int_{0}^{z}[-n+\lambda t] t^{1 /(n+1)-1} d t,
$$

or equivalently

$$
f^{\prime}(z)-(n+1) \frac{f(z)}{z} \prec-n+\frac{\lambda}{n+2} z .
$$

We can rewrite the relation (2.6) as

$$
f^{\prime}(z)-(n+1) \frac{f(z)}{z}=-n+\frac{\lambda}{n+2} \omega(z),
$$

for some $\omega \in B_{n}$. Here,

$$
\begin{gathered}
B_{n}=\left\{\omega \in \mathcal{H}: \omega(0)=\omega^{\prime}(0)=\cdots=\omega^{(n)}(0)=0,\right. \text { and } \\
|\omega(z)|<1 \text { for } z \in \Delta\} .
\end{gathered}
$$

If we consider

$$
g(z)=\frac{f(z)}{z}-1
$$

then the relation $(2.7)$ can be written as

$$
z g^{\prime}(z)-n g(z)=\frac{\lambda}{n+2} \omega(z) .
$$

An algebraic computation implies that

$$
g(z)=a_{n+1} z^{n}+\frac{\lambda}{n+2} \int_{0}^{1} \frac{\omega(t z)}{t^{n+1}} d t .
$$

As $\omega(z) \in B_{n}$, Schwarz's lemma gives that $|\omega(z)| \leq|z|^{n+1}$ for $z \in \Delta$ and therefore,

$$
|g(z)| \leq|z|^{n}\left(\left|a_{n+1}\right|+\frac{\lambda}{n+2}|z|\right), \quad z \in \Delta
$$


which is

$$
\left|\frac{f(z)}{z}-1\right|<\left|a_{n+1}\right|+\frac{\lambda}{n+2}, \quad z \in \Delta .
$$

So that

$$
\left|\frac{f(z)}{z}\right|>1-\left|a_{n+1}\right|-\frac{\lambda}{n+2}=: k .
$$

Also from (2.6) it follows that

$$
\left|f^{\prime}(z)-(1+n) \frac{f(z)}{z}\right|<n+\frac{\lambda}{n+2} .
$$

Combining these last two inequalities, we see that

$$
\begin{aligned}
k\left|\frac{z f^{\prime}(z)}{f(z)}-(1+n)\right| & <\left|\frac{f(z)}{z}\right|\left|\frac{z f^{\prime}(z)}{f(z)}-(1+n)\right| \\
& =\left|f^{\prime}(z)-(1+n) \frac{f(z)}{z}\right|<n+\frac{\lambda}{n+2}
\end{aligned}
$$

which simplifies to

$$
\left|\frac{z f^{\prime}(z)}{f(z)}-(1+n)\right|<\frac{n}{k}+\frac{\lambda}{(n+2) k} .
$$

This gives us

$$
\operatorname{Re}\left(\frac{z f^{\prime}(z)}{f(z)}\right)>\beta
$$

where

$$
\beta=\frac{(n+2)\left[1-(n+1)\left|a_{n+1}\right|-\lambda\right]}{(n+2)-(n+2)\left|a_{n+1}\right|-\lambda} .
$$

We introduce the following examples to illustrate Theorem 2.1:

Example 2.1. Let the function $f(z)=z+a_{n+1} z^{n+1}+a_{n+2} z^{n+2}$ be such that $(n+1)\left|a_{n+1}\right|+(n+2)\left|a_{n+2}\right|<1$. Then the function $f$ is starlike.

By putting $a_{n+1}=0$ and $\lambda=(n+2)(1-\gamma) /(n+2-\gamma),(0 \leq \gamma<1)$ in Theorem 2.1 we obtain

Example 2.2. The function $f(z)=z+\frac{(n+2)(1-\gamma)}{(n+2-\gamma)} z^{n+2},(0 \leq \gamma<1)$ is starlike of order $\gamma$.

Remark 2.1. We note that by using Theorem 2.1 we proved that Example 2.1 holds true, while from Theorem 1.1 it is not easy to show.

By making use of Theorem 2.1, we obtain the following result concerning the starlikeness of the double integral operator. 
Theorem 2.2. Let $c$ be a complex number with $|c|<1 /(n+1)$ and $0<\lambda \leq$ $1-|c|(n+1)$. Also suppose that $g(z) \in \mathcal{H}$ satisfies

$$
|g(z)|<\lambda,
$$

then the function $f(z)$ given by

$$
f(z)=z+c z^{n+1}+z^{n+2} \int_{0}^{1} \int_{0}^{1} g(r s z) s^{n+1} d r d s
$$

is starlike of order $\beta$ in $\Delta$, where $\beta$ is given by (2.2).

Proof. First let the function $f(z) \in \mathcal{A}_{n}$ be the solution of the differential equation

$$
z f^{\prime \prime}(z)-n\left(f^{\prime}(z)-1\right)=z^{n+1} g(z)
$$

then we have

$$
\left|z f^{\prime \prime}(z)-n\left(f^{\prime}(z)-1\right)\right| \leq\left|z^{n+1}\right||g(z)|<\lambda .
$$

Thus, from Theorem 2.1, we conclude that $f(z)$ is starlike of order $\beta$, where $\beta$ is given by (2.2). The solution of (2.8) can be obtained by integrating twice. By setting $\varphi(z)=f^{\prime}(z)-1$, we can rewrite the equation (2.8) as

$$
z \varphi^{\prime}(z)-\varphi(z)=z^{n+1} g(z)
$$

which has solution $\varphi(z)$ given by

$$
\varphi(z)=c z^{n}+z^{n+1} \int_{0}^{1} g(r z) d r .
$$

Since $\varphi(z)=f^{\prime}(z)-1$, we have

$$
f^{\prime}(z)-1=c z^{n}+z^{n+1} \int_{0}^{1} g(r z) d r
$$

that is

$$
f(z)=z+c z^{n+1}+z^{n+2} \int_{0}^{1} \int_{0}^{1} g(r s z) s^{n+1} d r d s
$$

Example 2.3. Let $c$ and $k$ be complex numbers such that $(n+1)|c|+|k|<1$. Then for the function $g(z)=k z$, we have

$$
f(z)=z+c z^{n+1}+\frac{k}{2(n+3)} z^{n+3}
$$

and this function is starlike. 
Example 2.4. Let $c$ and $k$ be complex numbers such that $(n+1)|c|+|k|<1$. Then for the function $g(z)=k$, we have

$$
f(z)=z+c z^{n+1}+\frac{k}{(n+2)} z^{n+2}
$$

and this function is starlike.

In the next results we generalize the results obtained in [4].

Theorem 2.3. Let $0 \leq \beta<1$ and $\alpha$ be a complex number with $\max \{0,|\alpha|+$ $\beta-1\} \leq$ Re $\alpha<n$ and let $f(z) \in \mathcal{A}_{n}$. If $f(z)$ satisfies

$$
\left|z f^{\prime \prime}(z)-\alpha\left(f^{\prime}(z)-1\right)\right|<\frac{(n-\operatorname{Re} \alpha)(n+1)[1+\operatorname{Re} \alpha-|\alpha|-\beta]}{n+1-\beta},
$$

then $f(z)$ is starlike of order $\beta$ in $\Delta$.

Proof. The inequality (2.9) can be written as follows

$$
z f^{\prime \prime}(z)-\alpha\left(f^{\prime}(z)-1\right) \prec \frac{(n-\operatorname{Re} \alpha)(n+1)[1+\operatorname{Re} \alpha-|\alpha|-\beta]}{n+1-\beta} z .
$$

If we set

$$
\begin{aligned}
p(z) & =f^{\prime}(z)-(1+\alpha) f(z) / z \\
& =-\alpha+(n-\alpha) a_{n+1} z^{n+1}+\ldots \in \mathcal{H}[-\alpha, n],
\end{aligned}
$$

then (2.10) becomes

$$
p(z)+z p^{\prime}(z) \prec-\alpha+\frac{(n-\operatorname{Re} \alpha)(n+1)[1+\operatorname{Re} \alpha-|\alpha|-\beta]}{n+1-\beta} z .
$$

Applying Lemma 1.1 as well as using the proof of Theorem 2.1, we conclude that

$$
p(z) \prec \frac{1}{n z^{1 / n}} \int_{0}^{z}\left(-\alpha+\frac{(n-\operatorname{Re} \alpha)(n+1)[1+\operatorname{Re} \alpha-|\alpha|-\beta]}{n+1-\beta} t\right) t^{1 / n-1} d t,
$$

or equivalently

$$
p(z) \prec-\alpha+\frac{(n-\operatorname{Re} \alpha)[1+\operatorname{Re} \alpha-|\alpha|-\beta]}{n+1-\beta} z .
$$

Hence from (2.11) it follows that

$$
f^{\prime}(z)-(1+\alpha) \frac{f(z)}{z} \prec-\alpha+\frac{(n-\operatorname{Re} \alpha)[1+\operatorname{Re} \alpha-|\alpha|-\beta]}{n+1-\beta} z .
$$

We can rewrite (2.12) as

$$
f^{\prime}(z)-(1+\alpha) \frac{f(z)}{z}=-\alpha+\frac{(n-\operatorname{Re} \alpha)[1+\operatorname{Re} \alpha-|\alpha|-\beta]}{n+1-\beta} \omega(z),
$$


for some $\omega(z) \in B_{n-1}$. If we consider

$$
g(z)=\frac{f(z)}{z}-1
$$

then $(2.13)$ can be rewritten as

$$
z g^{\prime}(z)-\alpha g(z)=\frac{(n-\operatorname{Re} \alpha)[1+\operatorname{Re} \alpha-|\alpha|-\beta]}{n+1-\beta} \omega(z) .
$$

An algebraic computation implies that

$$
g(z)=\frac{(n-\operatorname{Re} \alpha)[1+\operatorname{Re} \alpha-|\alpha|-\beta]}{n+1-\beta} \int_{0}^{1} \frac{\omega(t z)}{t^{\alpha+1}} d t .
$$

As $\omega(z) \in B_{n-1}$, Schwarz's lemma gives that $|\omega(z)|<|z|^{n}$ for $z \in \Delta$ and therefore

$$
|g(z)|<\frac{1+\operatorname{Re} \alpha-|\alpha|-\beta}{n+1-\beta}|z|^{n},
$$

which is

$$
\left|\frac{f(z)}{z}-1\right|<\frac{1+\operatorname{Re} \alpha-|\alpha|-\beta}{n+1-\beta}
$$

or

$$
\left|\frac{f(z)}{z}\right|>1-\frac{1+\operatorname{Re} \alpha-|\alpha|-\beta}{n+1-\beta}=\frac{n-\operatorname{Re} \alpha+|\alpha|}{n+1-\beta} .
$$

Also from (2.12) we obtain

$$
\begin{aligned}
\left|f^{\prime}(z)-(1+\alpha) \frac{f(z)}{z}\right| & <|\alpha|+\frac{(n-\operatorname{Re} \alpha)[1+\operatorname{Re} \alpha-|\alpha|-\beta]}{n+1-\beta} \\
& =\frac{(1+\operatorname{Re} \alpha-\beta)(|\alpha|+n-\operatorname{Re} \alpha)}{n+1-\beta} .
\end{aligned}
$$

Combining these two last results, we observe that

$$
\begin{aligned}
& \frac{n-\operatorname{Re} \alpha+|\alpha|}{n+1-\beta}\left|\frac{z f^{\prime}(z)}{f(z)}-(1+\alpha)\right|<\left|\frac{f(z)}{z}\right|\left|\frac{z f^{\prime}(z)}{f(z)}-(1+\alpha)\right| \\
& =\left|z f^{\prime}(z)-(1+\alpha) \frac{f(z)}{z}\right|<\frac{(1+\operatorname{Re} \alpha-\beta)(|\alpha|+n-\operatorname{Re} \alpha)}{n+1-\beta}
\end{aligned}
$$

which implies that

$$
\left|\frac{z f^{\prime}(z)}{f(z)}-(1+\alpha)\right|<1+\operatorname{Re} \alpha-\beta
$$

or

$$
\operatorname{Re}\left(\frac{z f^{\prime}(z)}{f(z)}\right)>(1+\operatorname{Re} \alpha)-(1+\operatorname{Re} \alpha-\beta)=\beta .
$$

and the proof is complete. 
We note that when in Theorem 2.3, $\alpha$ is a real number we get Theorem 1.1. In the same way is in the proof of Theorem 2.2 we obtain the following result, the details are thus omitted.

Theorem 2.4. Let a function $g(z) \in \mathcal{H}$ satisfy

$$
|g(z)| \leq \frac{(n-\operatorname{Re} \alpha)(n+1)[1+\operatorname{Re} \alpha-|\alpha|-\beta]}{n+1-\beta},
$$

for some $0 \leq \beta<1$ and $\max \{0,|\alpha|+\beta-1\} \leq$ Re $\alpha<n$. Then the function $f(z)$ given by

$$
f(z)=z+z^{n+1} \int_{0}^{1} \int_{0}^{1} g(r s z) r^{n-\alpha-1} s^{n} d r d s
$$

is starlike of order $\beta$ in $\Delta$.

\section{REFERENCES}

[1] R. Aghalary and A. Ebadian, New coefficient conditions for functions starlike with respect to symmetric points, Serdica Math. J., 36 (2010), 211-222.

[2] R. M. Ali, S. K. Lee, K. G. Subramanian and A. Swaminathan, A third-order differential equation and starlikeness of a double integral operator, Abstr. Appl. Anal., (2011), Art. ID 901235, 10 pp.

[3] D. J. Hallenbeck and St. Ruscheweyh, Subordination by convex functions, Proc. Amer. Math. Soc., 52 (1975), 191-195.

[4] K. Kuroki and S. Owa, Double integral operators concerning starlike of order $\beta$, Int. J. Differ. Equ., (2009) 1-13.

[5] S. S. Miller and P. T. Mocanu, Double integral starlike operators, Integral Transforms Spec. Funct., 19 (7-8) (2008), 591-597.

[6] M. Obradović, Simple sufficient conditions for univalence, Mat. Vesnik, 49 (1997), 241-244.

[7] J. Sokól, On sufficient condition for starlikeness of certain integral of analytic function, J. Math, Appl., 26 (2006), 127-130.

[8] J. Sokól, On a condition for $\alpha$-starlikeness, J. Math. Anal. Appl., 352 (2009), 696-701.

(Received: May 30, 2013)

\author{
Rasoul Aghalary \\ Department of Mathematics \\ Faculty of Science \\ Urmia University \\ Urmia, Iran \\ raghalary@yahoo.com \\ Santosh Joshi \\ Department of Mathematics \\ Walechand college of Engineering \\ Sangli, India \\ joshisb@hotmail.com
}

